\title{
Effects of Physical Rotational Movement Difference and Handwriting Position on Academic Achievement and Learning Disabilities
}

\author{
Rowe A. Young* \\ Department of Psychology Program/Behavioral Neuroscience, University of Connecticut Storrs, Storrs, USA \\ Email: Roweyk@gmail.com
}

Received 27 January 2015; accepted 11 February 2015; published 12 February 2015

Copyright (c) 2015 by author and Scientific Research Publishing Inc.

This work is licensed under the Creative Commons Attribution International License (CC BY). http://creativecommons.org/licenses/by/4.0/

\section{Abstract}

Only recently has movement behavior and learning disabilities (LD), including the confusing topic of dyslexia, been considered to be connected. The realization that phonemic problems may often be the "result" of learning issues such as dyslexia, and not the cause, has finally surfaced. This condition is hypothesized as being primarily due to not sensing the sound connection because of the hand feeling the shape of feeling letters incorrectly. This consequently, interferes with the sound value(s) being connected during the learning process. We herein provide the results of an earlier unpublished study, which demonstrates how we obtained these and other classroom results. We provide evidence of movement difference between those with identified LDs and those without LDs, and how this can relate to both left and inverted rotational direction difference. Other movement differences are also identified as modifiers of the achievement difference between the students found in the at-risk populations. As previously described (Young et al., 2012), understanding "reversed positioning sensation" (RPS) can be of great assistance to those with the basic condition known as written language disability (WLD) or dysgraphic conditions, which are often recognized entities of the LD known as dyslexia. This is important, because remediation involves changing the hand position of those affected and is theorized, can change the way the brain senses the direction of learning (i.e., to make letters by processing the sense of feeling them top/down instead of from an inverted sensation of sensing bottom/up movement). That this remedial movement concept is so amazingly simple may be why it has been overlooked for years.

\section{Keywords}

Learning Disability, Rotational Movement, Handwriting, Dyslexia, Academic Achievement

\footnotetext{
*Retired.
} 


\section{Introduction}

It is accepted that many factors contribute to learning disabilities (LDs), including visual difference (YoungKaple, 2013). LDs, citing dyslexia in particular, may also result from distinct cognitive impairments characterized by distinguishable anatomical markers (Jednoróg et al., 2014). Originally, the word dyslexia (LD) was created to imply being backward or reversing letters (Orton, 1928; Wagner, 1973). This, however, was almost solely attributed to mirroring due to the visual system and hemispheric dominance interaction. While no specific LD is diagnosed in the reported study presented here, that left and inverted movement preference are identified in the learning disabled found here as in previous studies (Young \& Ginsburg, 1993), suggests, that the markers indicating left side and or inverted rotational behavior, that might be identified with the dyslexia syndrome, are present.

Recently, studies utilizing functional magnetic resonance imaging, have identified a neurological movement connection to dyslexia (Waldie et al., 2013). According to Waldie and colleagues, "Our participants with dyslexia also showed over activation in the right hemisphere regions of the putamen and precentral gyrus during both regular and pseudo word lexical decision making. The putamen comprises part of both the dorsal striatum and the basal ganglia and is connected to the substantia nigra and globus pallidus". "Though the putamen is thought to have no specific specialization, it is involved in regulating movements and implicit learning (Packard $\mathrm{M}$ et al., 2002)". Waldie and colleagues further state: "The precentral gyrus is also associated with initiating the onset of movements. It is likely that this activity reflects increased reliance on silent articulatory processes. Other studies are consistent with this finding with dyslexic over activation in precentral/motor regions during phonological reading tasks.” (Wimmer et al., 2010) Another recent study provided empirical support for the view that a letter-speech sound-binding deficit is a key factor in dyslexia (Aavena et al., 2013).

How hand use and positioning impacts learning and achievement has been the focus of our ongoing investigation (Young et al., 1987, 1993, 1995, 2006, 2008, 2012). This interest resulted from our observations that many persons with a history of LDs were found to employ atypical approaches to performing simple tasks, such as bimanually opening a bottle. This involves using the hand that grips the bottom of the bottle to turn and open the bottle, rather than holding the bottom of the bottle while turning the top. We named this condition "Reversed Positioning Sensation" (RPS). By comparing differences in the preference of hand positions, we found certain movement behavior to be predictive of academic achievement and aptitude difference. It has been hypothesized that an individual's fine and gross motor rotational and hand positioning preference affect both learning and academic achievement (Young, 2004, 2006, 2008). Originally, to help us to investigate these behavioral differences, we devised a test called the Young-Ginsburg Lateral Direction Assessment \& Spatial Test (YGLD) (Young \& Ginsburg, 1987, 2011). As a result of our investigations of more than 1100 subjects, it is further suggested that interceding and encouraging adoption of successful, remedial hand repositioning (especially in preschool and the early grades) could significantly remediate academic performance. In this paper, we present evidence demonstrating the physical rotational movement difference between high and low achievers found in a normal fifth grade population. We also compare handwriting differences to bimanual rotation differences.

\section{Methodology}

To assess the effects of movement and handwriting position on classroom achievement, the following methods, using an opportunistic elementary public school population, were employed. Requests were sent to the parents of a population of fifth grade students at a suburban Connecticut elementary school. We requested parental permission to access school records and for participation in our study. This homogeneous population was selected to help minimize environmental differences within the sample. Fifty students from five different classrooms were given permission by their parents to participate. There were $n=21$ females and $n=29$ males. The average age of the students was eleven years of age. The group ranged in age from ten though eleven years and eight months. The parents were also asked to fill out questionnaires regarding medical histories involving visual, hearing or motor impairments. One student was diagnosed as having muscular dystrophy; it was decided that his direction of movement was not impaired so this student was included. There were 6 students with myopia and 1 with amblyopia; all 7 participated. No hearing impaired students were identified. While we were apprised that there were LD-diagnosed students in the group, we did not know how many were LD, or the specific LD-related diagnosis.

School records were employed to obtain scores from the IOWA Tests of Basic Skills (IOWA) and the Cognitive Aptitude Test (CAT). All administrative and testing personnel were blinded to student records and parental questionnaires before and during data collection. School records, parental questionnaires, and student interviews were also employed to identify early learning problems and problems with continuity of handedness, and for any 
change of writing handedness (left-handed [LH] or write-handed [RH]). We identified 2 males and 1 female who were LH, but had been switched to being RH. These students were included in the study since we found from our prior investigations that writing handedness was not considered a necessary criterion for determining dominant hand rotation behavior (Young, 1989, 1993). There were two students found who presently used their $[\mathrm{LH}]$ left hand for writing in the sample.

The YGLD test was administered to all 50 students. Three of the YGLD sub-tests were employed for the current analysis: Test 1: direction preference for bimanual rotation of both fine and large motor movements of the hands (bimanual movement rotation test); Test 2: fine motor lateral direction consistency (curly line drawing test); and Test 3: the writing hand position. Only items relating to this present report will be described herein.

Three testing stations were set up; one for each section of the test. Students came to our testing room in groups of 3. Each student completed parts of the assessment at each station and then moved to the next station. This was to help prevent any confounding bias resulting from station-to-station performance.

Student IOWA and CAT test scores were converted to mean national predicted percentile rank scores for analytic simplicity. The sample's total reading mean was found to be the same or better than 79th percentile rank of Grade 5 students nation-wide. Their language, math and overall achievement skills were at the 84th, 85th, and 84th percentiles, respectively. The standard score for the age group was at the 83rd national percentile rank.

Independent variables from each sub-test were used to group students within each sub-test as follows: Test 1. Bimanual movement rotation test (4 groups: right, either, left, or inverted bimanual turning); Test 2. Curly line drawing test (2 groups: consistent or inconsistent movement); and Test 3. Hand writing irregularities test (2 groups: regular or irregular hand position). Test methods and criteria for each sub-test are described below.

\section{Test 1. Bimanual movement rotation test}

A small bottle with a 1-inch diameter cap is employed for this test. There are 4 possible sub-group designations based on the students preferred hand position and motoric preferences: 1) Right bimanual: exclusive right turning hand on the top with a stationary hold on the bottom; 2) Either bimanual: turns both right and left hand on the top with a stationary hold or tandem movement on the bottom; 3) Left bimanual: exclusive left turning hand on top, with either no bottom right hand movement or right hand movement in tandem with the top left hand; 4) Inverted bimanual: either left or right hand stationary on top and turning with the bottom hand. This can be used in conjunction with other comfortable bottle opening techniques.

The student is first asked to unscrew the lid from the bottle and the examiner records which method was used. Next, the examiner demonstrates different approaches for unscrewing the lid of the bottle and the student is asked to repeat each approach. Only the technique(s) that appears natural and comfortable to the student, and that the student says that they would use, is recorded.

\section{Test 2. Curly line drawing test}

The curly line drawing test observes how the left and right hands prefer to move and rotate. This activity consists of drawing a continuous connected looped line of circles starting from an " $\mathrm{X}$ " placed in the middle of a line; the choice is given to start from the right of the " $\mathrm{X}$ " or from the left of the " $\mathrm{X}$ ". The student is instructed to first use the right hand to draw a curly line with loops in it. They are instructed to start on either side (but just one side of the " $X$ "); this is then repeated using the left hand on the next line. The test has 8 possible outcomes or combinations. The eight outcomes are divided into 2 groups: consistent or inconsistent. In the consistent group, both the LH and RH rotational movements are in the same direction, i.e., both are in either the clockwise or counter-clockwise direction and both hands will move in the same lateral direction. The consistent group will use the same side of the $\mathrm{X}$ with both hands, and will move in the same rotational direction. In the inconsistent group, the RH and the LH do not rotate in the same direction and/or the right and left hand does not move in the same lateral direction.

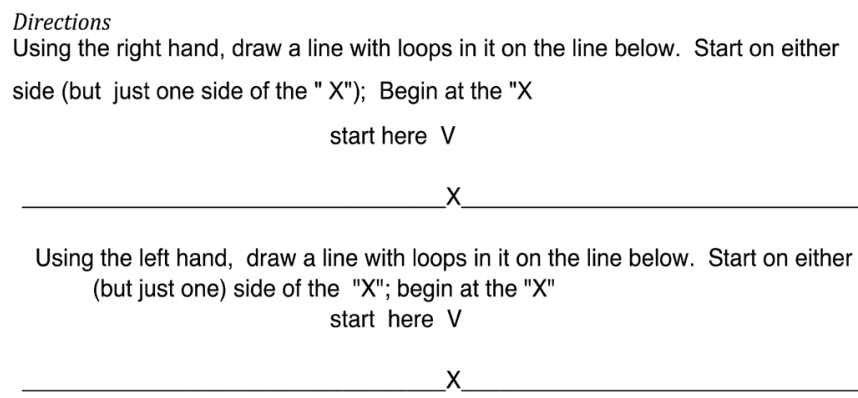




\section{Test 3. Handwriting irregularities test}

For this part of the study, only 2 groups (exclusive left top and inverted bimanual rotation) were used since these were the only groups with LD-identified students.

Handwriting irregularities are defined by how the writing hand is positioned (i.e., how the fingers are positioned to direct the pencil). If the pencil is placed between the thumb and index finger and resting upon the middle finger when writing, and the hand is resting on its side, this position is considered normal. Any other position is classified as irregular. Turning the wrist so that it is twisted or in a hooked position might also be observed and marked as irregular. Each irregular characteristic, as described below, was then checked off on the test sheet.

Directions for the handwriting irregularity test

Mark toward which hand the top of the writing paper is turned when the student writes with the writing hand. a. right b. left c. either d. does not turn

Is the writing hand inverted (bent, hooked) when writing? a. yes b. no

Is the pencil place between the thumb and index finger and resting upon the middle finger when writing? a. yes_b. bo no

When writing, is the hand resting on its side, on the paper? a. yes b. no

\section{Results}

Student performance on 3 motor tests (bimanual rotation test, curly line drawing test, and hand writing irregularities test correlated with academic performance on their Iowa test and CAT scores. Additionally, select items from the bimanual rotation test were highly predictive of a student being classified as LD.

Test 1. Bimanual movement rotation test

Figure 1 and Figure 2 present IOWA \& CAT scores plotted for the 4 subgroups identified by the bimanual rotation test (inverted, exclusive left top, either top, and exclusive right top). Analysis of variance (ANOVA) was calculated on the "language total" (LANGTOT) scores from the IOWA test and on the Composit (COMPOSIT) scores on the CAT. For LANGTOT, there were statistically significant differences among the 4 groups, F $(3,49)$ $=4.78, p<0.01$. Post-hoc Tukey $\alpha$ tests revealed that the inverted and exclusive left top groups had LANGTOT means that were significantly lower than those in the either top or exclusive right top groups. However, students in the inverted and exclusive left top groups did not differ from each other, nor did those in the either top or exclusive right groups. For COMPOSIT on the CAT test, there were also statistically significant differences among the 4 groups, $\mathrm{F}(3,46)=3.60, p<0.05$. Post-hoc Tukey $\alpha$ tests showed that the inverted group has a significantly lower COMPOSIT mean than any of the other 3 groups. Also, the exclusive right top group had a higher mean than any of the other 3 groups.

Additional analysis was undertaken to determine the relationship between bimanual rotation preference and LD. No LD students were identified in the exclusive right top $(\mathrm{n}=20)$ or the either bimanual $(\mathrm{n}=9)$ rotation groups. All 7 LD students were located in the left bimanual $(n=4)$ and inverted bimanual $(n=3)$ subgroups. A chi squared analysis $\left(\chi^{2}=11.42, d f=3, p=0.01\right.$. Cramer's $\mathrm{V}=0.47$ ) indicated a statistically significant difference between these observed frequencies and what would be expected by chance. In other words, there is a nonrandom (moderately strong) association between LD status and hand rotational preference.

Analysis of rotational preference and LD designation revealed $\chi^{2}=7.29, d f=3, p=0.06$. The chi-square test was just below the threshold for statistical significance, indicating that the LD group had no systematic preference for any given rotational pattern. However, examination of the observed frequencies suggests that LD participants displayed either an inverted rotation or an exclusive left top rotation, to the exclusion of the other 2 rotational preferences. Larger sample sizes are necessary to address this suggestion statistically. With 43 non-LD students and 7 LD students, it is unlikely that the sample of LD students was sufficient to conclusively document an association between LD status and rotational preference. However, these findings suggested that non-LD students were more inclined toward exclusive top right rotation or either top, whereas LD students were more inclines toward either inverted or exclusive top left rotation $(p=0.002)$.

There were systematic differences in some students who demonstrated a preference for inverted or left bimanual rotation but were not found to have noticeable achievement difficulties. The inverted bottom $(\mathrm{n}=11)$ and the left top $(\mathrm{n}=10)$ rotation hand preference groups had significantly lower achievement and aptitude scores than right top $(\mathrm{n}=20)$ or either top $(\mathrm{n}=9)$ preferring groups. All LD students $(\mathrm{n}=7)$ were found in the inverted or left rotation groups. 


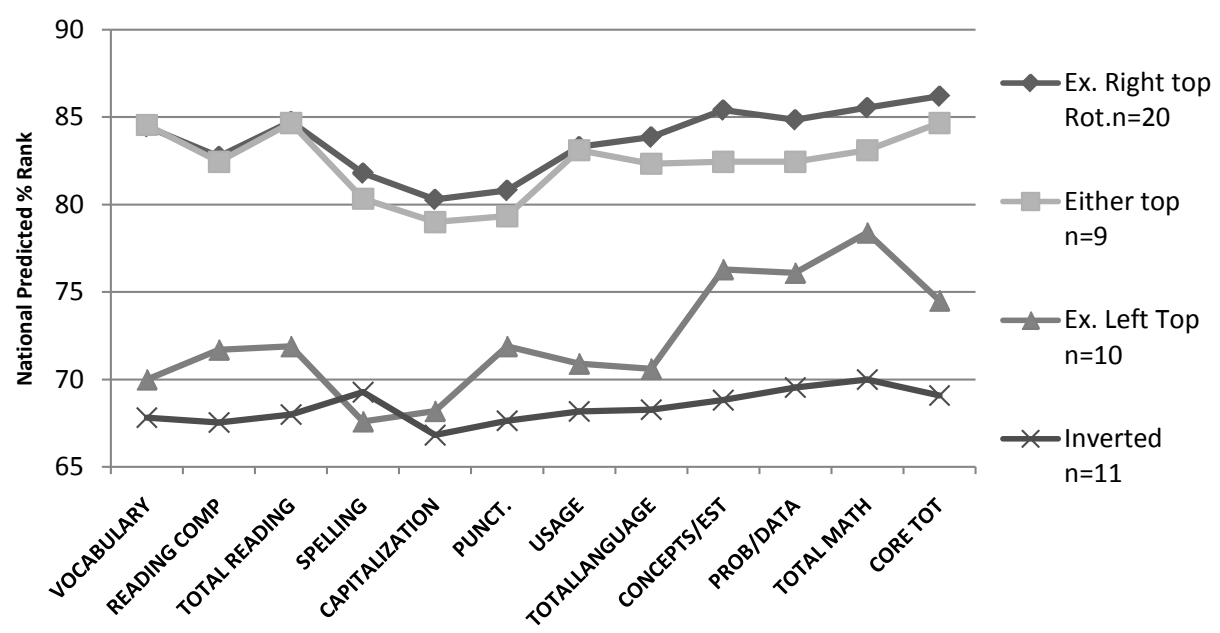

Figure 1. Bimanual rotation groups compared with IOWA test results.

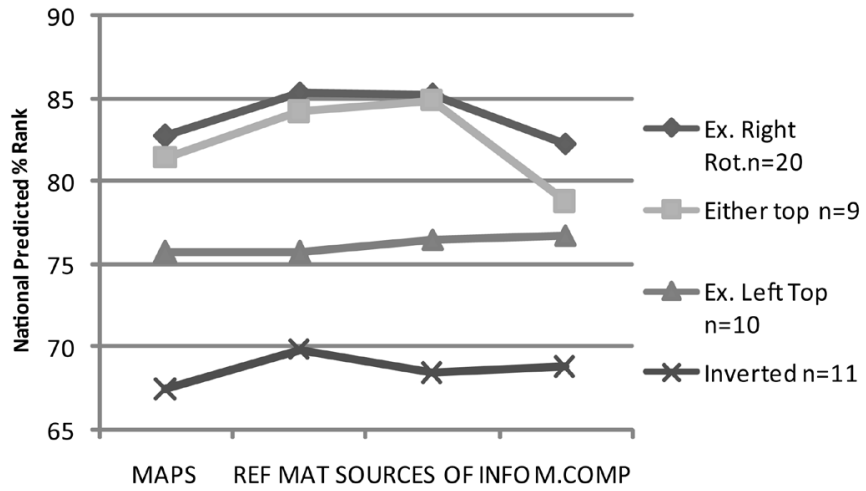

Figure 2. Bimanual rotation groups compared with CAT cognitive aptitude test results.

\section{Test 2. Curly line drawing test compared to IOWA test results}

Data from the curly line drawing test shows division of the total student population between consistent and inconsistent motor direction (Figure 3). Consistency was defined as moving BOTH hands in the same lateral direction from the starting point $(\mathrm{X})$ AND using a consistent turning rotational direction (either clockwise or counter clockwise) with BOTH hands. The consistent $(\mathrm{n}=28)$ and inconsistent $(\mathrm{n}=22)$ rotation groups scored in the 80th and 70th percentiles, respectively, on the IOWA test. When we tested for consistency of bilateral fine motor movement, we found that the consistent group scored significantly higher in both Total Language ( $p=$ $0.007)$ and Core Total on the IOWA test subsets $(p=0.009)$ compared to the inconsistent group. One LD student was identified in the consistent rotation grouping.

\section{Test 3. Handwriting irregularities test}

Figure 4 shows the association of hand writing position with select standardized tests. Eight students who were observed to use an irregular hand writing pencil grip were found in the exclusive left and inverted bimanual rotation groups. IOWA achievement scores were significantly higher $(p=0.001)$, than this group's "regular" position IOWA achievement scores (Figure 4). All7 LD students were found in the inverted bimanual or exclusive left top rotation groups $\left(\chi^{2}=11.42, d f=3, p=0.01\right)$. Cramer's $\mathrm{V}=0.47$. This indicates a significant difference between these observed frequencies and what would be expected by chance. In other words, there is a nonrandom association between LD status and hand rotational preference. This is a moderately strong association. Yates corrected $\chi^{2}=6.90, d f=1, p=0.01$. Because of the small cell sizes, the Yates correction was used on this chi square test, indicating statistical significance (i.e., the distributions of regular and irregular hand writing positions are not random, differing significantly from what would be expected by chance alone). The Cramer's V measure of association is 0.67 , which is fairly strong. This implies that group membership (LD, non-LD) has a substantial association with hand writing position. Obviously, that association is most evident among those 
CONSISTENT CURLY LINE DRAWING COMPARED TO INCONSISTENT CURLY LINE DRAWING COMPARED TO IOWA TEST

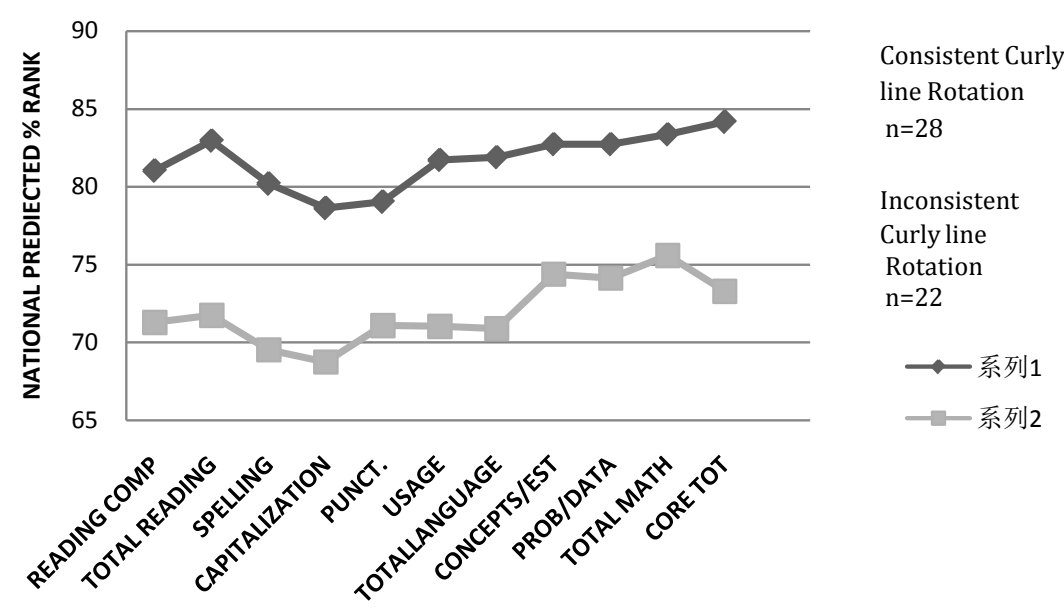

Figure 3. Curly line drawing test compared to IOWA test results.

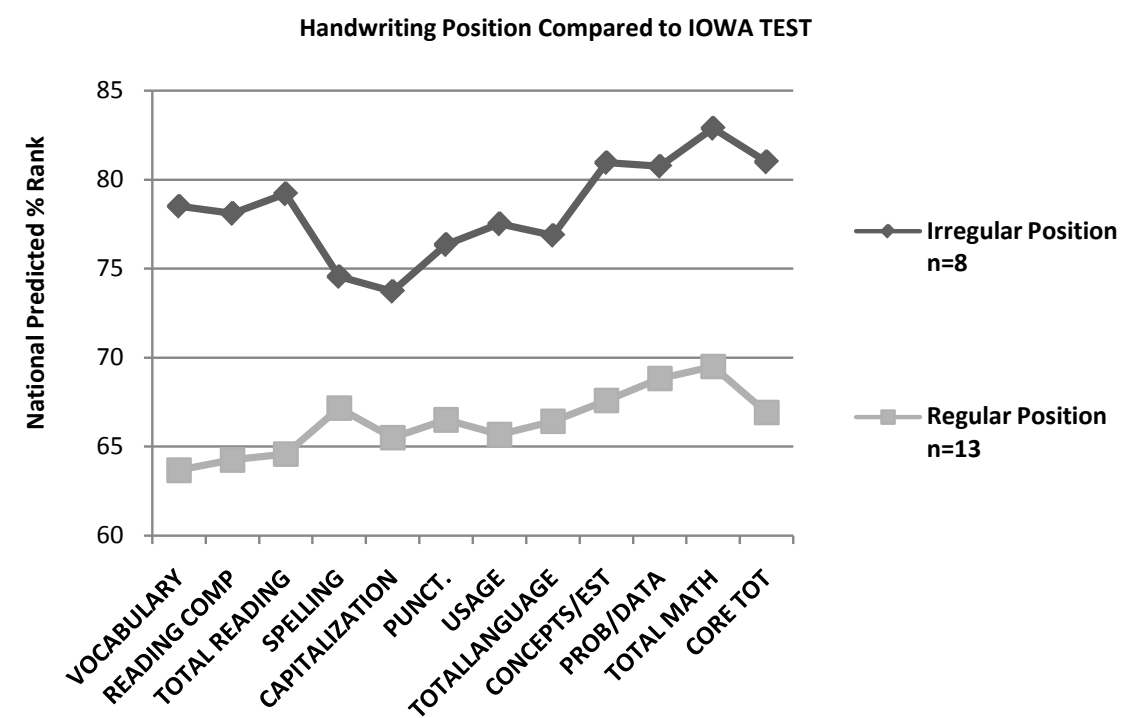

Figure 4. Comparison of unusual and normal handwriting positions with IOWA test results in students with regular or irregular writing positions (combined exclusive left and inverted rotational groups).

with an irregular hand writing position. This is where the non-LD students were identified and the LD students were not identified. With a sample of only 21 students, these results would need to be replicated but these findings are at least suggestive of an association between LD status and hand writing position.

\section{Discussion}

Using motor and movement characteristics, we were able to isolate all 7 LD-identified students into 2 of the bimanual (exclusive left and inverted) rotational subgroups. Three other characteristics were found in the non-LD students from the exclusive left and inverted rotation groups $(n=14)$ that were not found in the LD group $(n=7)$ : handwriting irregularities $(\mathrm{n}=8)$, consistency in the curly line drawing test $(\mathrm{n}=7)$, and clockwise movement with the writing hand $(n=4)$. While it is only suggestive that 4 of the students, all of whom were non-LD, used a clockwise movement writing hand direction. Clockwise movement naturally produces a top/down movement. None of the participants in the exclusive right $(\mathrm{n}=20)$ or either bimanual $(\mathrm{n}=9)$ groups were found with this movement characteristic. The non-LD students in this division who did not have a handwriting difference had at 
least 1 of these other movement differences; the only exception was 1 of the 6 non-LD females in the division. An LD male in the exclusive left and inverted grouping was found to have consistency on the curly line drawing test. This finding, however, was questionable since the student's work differed from the others in this test. His curly lines were not drawn on the lines of the paper as instructed; instead, they were drawn as matching curls in an upwards fashion, away from the line.

There were only 3 females in the left top and 3 females in the inverted group; none were identified as LD. In a previously reported study (Young \& Ginsburg, 1993), we identified 8 female left top rotators, none of whom were identified as LD. In contrast, in the same study, we identified 9 male left top rotators, 7 of whom were identified as being LD. In the present study, all 7 LD-identified students were male and in the exclusive top left and inverted bimanual groups. While suggestive, it is still unclear whether or not a gender difference related to this particular bimanual rotation laterality exists; new research identifying female dyslexia may contribute to this finding (Evans et al., 2013).

The 28 students found in all rotational groups who were found to be consistent on the curly line drawing test produced significantly higher achievement scores being in the 80th percentiles than for the inconsistent rotators $(n=22)$, whose achievement scores were in the 70th percentiles on national percentile-predicted rank scores.

It is also important to note, that when studying other school groups that were tested in the same manner, exclusive right top bimanual rotators have been identified as LD. In those studies, subjects identified as LD and right rotators have had significantly lower achievement scores compared with left and inverted LD subjects in the same classes. A reason other than a motor (movement) difficulty was suspected (Young et al., 1993).

\section{Conclusion}

This study demonstrates significant differences in student achievement (on IOWA and CAT tests) when comparing groups based upon the hand movement differences we have examined. Results of the bimanual rotation preference test demonstrated that students in both the inverted and exclusive left top rotating groups scored significantly lower academically than students in the exclusive right top or either top bimanual rotator groups. Results from the curly line drawing test demonstrated that students in all groups who are consistent in how they move both directionally and rotationally with both the right and the left hand scored significantly higher on academic tests. Results from the hand writing irregularities test demonstrates that students who used unusual handwriting positions, and were in the exclusive left top and bimanual inverted rotation preference groups, had higher academic achievement compared to those students without unusual writing positions in those same groups. One might describe this as using a "remedial grip". This is accomplished by turning the hand over, so the palm is flat with writing surface and holding the pencil between index and middle fingers. The fingers, are individually placed, so as to sense a top/down feeling for letters.

A key question arising from this research is, can this suspected hemispheric difference of exclusive left top or inverted rotator grouping be a major factor causing many of the symptoms of various identified LDs. The relationship found here between movement behavior and academic achievement here suggests a primary cause for the LD identification. Most importantly, with identification of these invisible but physical reversal tendencies possibly affecting as many as $20 \%$ of LD students found in many classroom populations (Torgesen, 2002). Interestingly, this is similar to the proportion of students that we have often blindly identified as being LD with the use of these reported behavioral and physical movement markers (Young et al., 1993; Young, 2004, 2006). It would now seem possible that proper handwriting "movement" changes could correct the tendency for a suspected difficulty such as "bottom/up" (RPS) sensation. New evidence from fMRI research provides the possibility that re-patterning and correcting handwriting movement to connect the correct physical shape of letters to their sound values, could properly retrain the mind. This investigation further suggests that interceding and encouraging the adoption of successful, remedial hand repositioning and handwriting training, (especially in preschool and the early grades) could significantly remediate academic performance.

Further research is necessary to replicate our findings and validate the utility of our proposed remedial intervention.

\section{Acknowledgements}

The author would like to thank Chris Segrin, PhD, University of Arizona for assistance with statistical analysis; and Penny Baron, MS and James Kaple PhD for editorial assistance in the preparation of this manuscript. 


\section{References}

Aavena, S., Snellings, P., Tijms, J., \& van der Molen, M. W. (2013). A Lab-Controlled Simulation of a Letter-Speech Sound Binding Deficit in Dyslexia. Journal of Experimental Child Psychology, 4, 691-707.

Evans, T. M., Flowers, D. L., Napoliello, E. M., \& Eden, G. F. (2013). Sex-Specific Gray Matter Volume Differences in Females with Developmental Dyslexia. Brain Structure Function, 219, 1041-1054. http://dx.doi.org/10.1007/s00429-013-0552-4

Jednoróg, K., Gawron, N., Marchewka, A., Heim, S., \& Grabowska, A. (2014). Cognitive Subtypes of Dyslexia Are Characterized by Distinct Patterns of Grey Matter. Brain Structure Function, 219, 1697-1707. http://dx.doi.org/10.1007/s00429-013-0595-6

Orton, S. T. (1928). Specific Reading Disability—Strephosymbolia. JAMA, 90, 1095-1099. http://dx.doi.org/10.1001/jama.1928.02690410007003

Packard, M., \& Knowlton, B. (2002). Learning and Memory Functions of the Basal Ganglia. Annual Review of Neuroscience, 25, 563-593. http://dx.doi.org/10.1146/annurev.neuro.25.112701.142937

Torgesen, J. K. (2002). The Prevention of Reading Difficulties. Journal of School Psychology, 40, 7-26. http://dx.doi.org/10.1016/S0022-4405(01)00092-9

Wagner, R. (1973). Rudolf Berlin: Originator of the Term Dyslexia. Ann Dyslexia, 23, 57-63.

Waldie, K. E., Haigh, C. E., Badzakova-Trajkov, G., \& Kirk, B. J. (2013). Reading the Wrong Way with the Right Hemisphere. Brain Sciences, 3, 1060-1075.

Wimmer, H., Schurz, M., Sturm, D., Richlan, F., Klackl, J., Kronbichler, M., \& Ladurner, G. (2010). A Dual-Route Perspective on Poor Reading in a Regular Orthography: An fMRI Study. Cortex, 46, 1284-1298. http://dx.doi.org/10.1016/j.cortex.2010.06.004

Young, R. A. (1989). Preferred Directional Rotational Motor Movement of the Fingers and Hands Compared to Reported Receptive/Expressive Written Language Ability. Unpublished Thesis, Storrs: University of Connecticut.

Young, R. A. (2004). Laterality in Kinesthetic Rotation of Movement Orientation and Visual Dominance Interaction as Mediators of Consciousness; Toward a Science of Consciousness. The Consciousness Conference: Toward a Science of Consciousness (pp. 46-47). Abstract \#92, University of Arizona, Tucson.

Young, R. A. (2006). Tested Lateral Visual and Motor Behavior Interaction Identify Suspected Aspects of Dyslexia. Behavior Genetics, 36, 952-990.

Young, R. A. (2008). Lost in Space. “Inverted Positioning Sensation” (IPS): As of Yet Unidentified Contributing Cause for ADHD and/or LD. The Consciousness Conference (p. 95). Abstract \#121, University of Arizona, Tucson.

Young, R. A., \& Ginsburg, B. E. (1987). Genetic Variations in Motor and Cognitive Patterns Associated with Reading Disabilities-Diagnosis and Remediation. Behavior Genetics, 17, 644.

Young, R. A., \& Ginsburg, B. E. (1987, 2011). Young-Ginsburg: Lateral Direction Assessment and Spatial Test; "YGLD” Storrs, CT.

Young, R. A., \& Ginsburg, B. E. (1993). The Directional Motor Link in Reading Disabilities. In Temporal Information Processing in the Nervous System: Special Reference to Dyslexia and Dysphasia. Annals of the New York Academy of Sciences, 682, 436-439. http://dx.doi.org/10.1111/j.1749-6632.1993.tb23016.x

Young, R. A., \& Ginsburg, B. E. (1995). Visuo-Motor Patterns in Dyslexia. Behavior Genetics, 25, 294.

Young, R. A., Ginsburg, B. E., \& Bradway, D. (2012). Physical and Behavioral Markers Help Identify Written Language Disability (WLD) Related to Attention Deficit Hyperactivity Disorder (ADHD). Psychology, 3, 36-44. http://dx.doi.org/10.4236/psych.2012.31006

Young-Kaple, R. A. (2013). Eye Dominance Difference Connection to LD Learning Disabilities. World Journal of Psychology Research, 1, 1-9. 
Scientific Research Publishing (SCIRP) is one of the largest Open Access journal publishers. It is currently publishing more than 200 open access, online, peer-reviewed journals covering a wide range of academic disciplines. SCIRP serves the worldwide academic communities and contributes to the progress and application of science with its publication.

Other selected journals from SCIRP are listed as below. Submit your manuscript to us via either submit@scirp.org or Online Submission Portal.
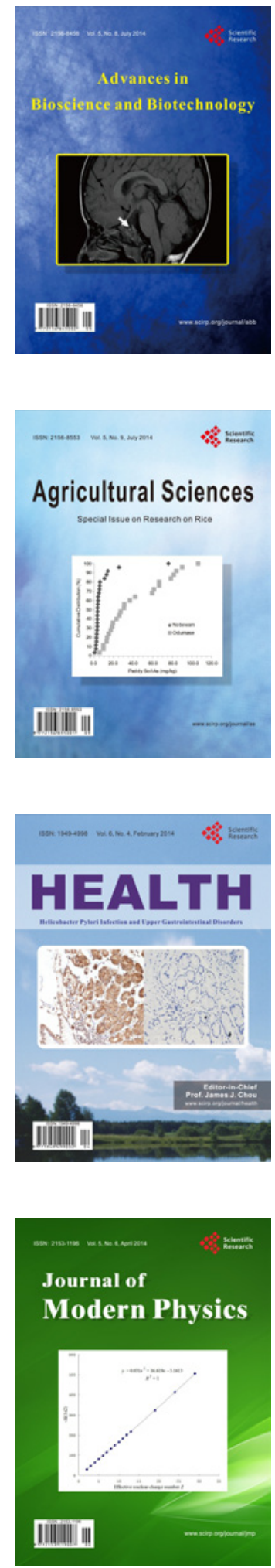
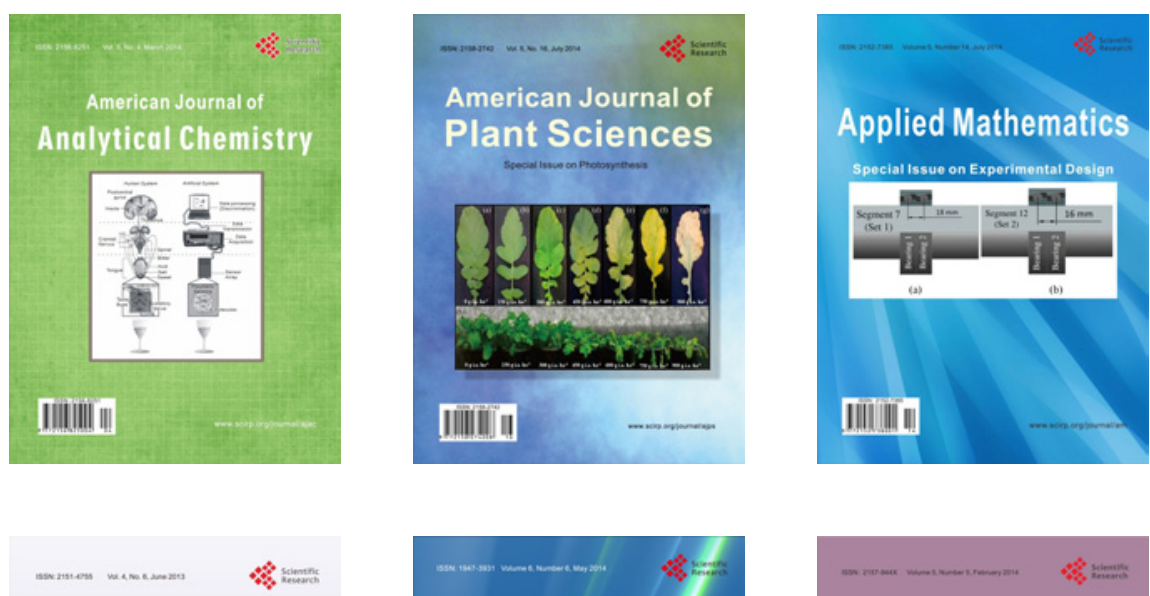

Creative Education
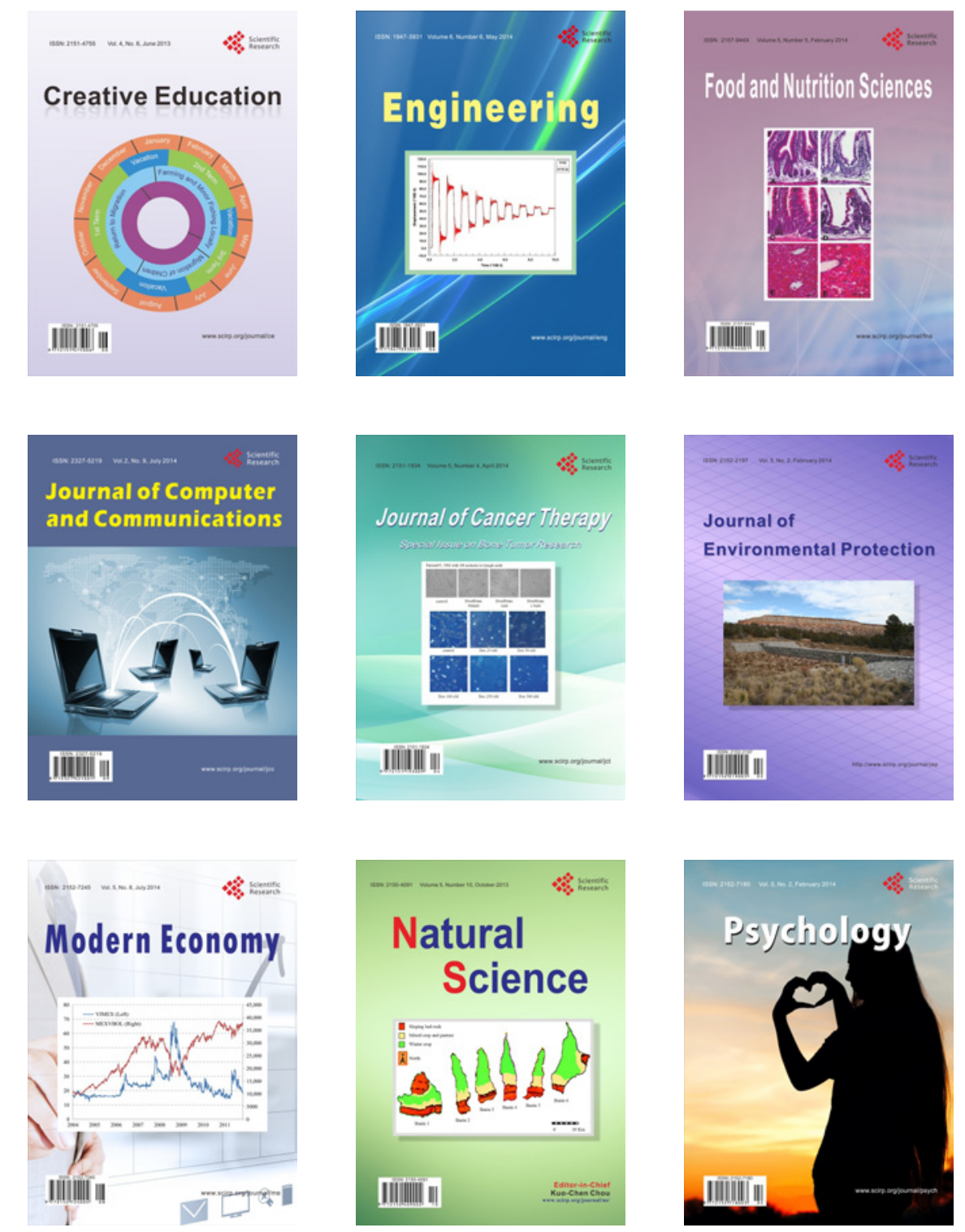